\title{
Light Curves of Supernovae
}

\author{
By BRUNO LIEBUNDGUT $\nmid$
}

Department of Astronomy, University of California

Berkeley, CA 94720, USA

\begin{abstract}
Although emerging from a range of progenitor stars and the product of different explosion mechanisms the light curves of the various supernova types are shaped mainly by radioactive power. Core-collapse supernovae have in addition early peaks from shock breakout with a subsequent cooling phase and massive extended stars a recombination (plateau) phase. Variations occur mostly due to differences of the progenitor stars. While there appears to be a fair understanding of the light curves of SNe II, new wrinkles are emerging for SNe Ia. The photometry of $\mathrm{SNe} \mathrm{Ib}$ and SNe Ic remains unsatisfactory.
\end{abstract}

\section{Introduction}

The temporal brightness variation of supernovae ( $\mathrm{SNe}$ ) as measured by photometry contains valuable and unique information on the evolution of the progenitor star and the explosion event. Combined with optical spectroscopy broad-band light curves have been the main tools for supernova investigations in the past (e.g. Minkowski 1964, Woosley \& Weaver 1986, Wheeler \& Harkness 1990, Kirshner 1990). The light curves are shaped by the size and mass of the progenitor star, various processes within the explosion itself, the radioactive ashes, and, in certain cases, the local environment.

Accurate photometry is mandatory to disentangle the physics driving the emission and the colors provide information on the temperature evolution. Telltale deviations from blackbody emission arise from the effects of the rapidly expanding atmosphere. The decline rates at different epochs and for supernovae of different types are indicative of the power sources, the explosion energy, and the envelope mass. Long after the explosion the light curves probe energy inputs from pulsars, long-lived radioactive isotopes, and shock-interaction with circumstellar material. Finding photometric differences between and within classes of supernovae hinges on the accuracy of the observations, and has hampered such investigations. Finally, photometry is needed for the use of supernovae as distance indicators. The expanding photosphere method (Schmidt et al. 1992) rests on reliable color and magnitude information. The hypothesis of SNe Ia as standard candles can be tested with good photometry only.

Obtaining accurate photometric observations of supernovae is not a trivial pursuit. Among the more common problems are unfavorable weather conditions hampering a detailed sampling of the light curve, the disappearance of the object behind the sun for extended periods of time, the complicated brightness structure of the background, combination of different data sets acquired by different observers at different telescopes and with different equipment, and the faintness of most objects. A case in point is SN 1987A, circumpolar for many southern observatories, for which large homogeneous data sets have become available sampling the light curves all year round (e.g., Bouchet et al. 1991, Suntzeff et al. 1991). The possibility to construct a bolometric light curve for SN 1987A has proven essential to compare the observations with detailed theoretical models (c.f. Arnett et al. 1990, McCray 1993 for reviews).

$\dagger$ Present Address: European Southern Observatory, Karl-Schwarzschild-Strasse 2, D-8046 Garching bei München, Germany 
Large compilations of broad-band photometric data have been collected and are the basis for comparative studies (Young \& Branch 1989, Leibundgut et al. 1991c, Patat et al. 1993). Such atlases have proven useful while uncovering the limitations of old data at the same time. The addition of new high-quality light curves is highly desirable and is under way at several observatories.

In the following we will first discuss the light curves of type II supernovae ( $\S 2$ ), before concentrating on SNe Ia ( $(3)$. The state of photometry on $\mathrm{SNe} \mathrm{Ib} / \mathrm{c}$ will briefly be described in section 4 . Conclusions are presented in the last section ( $\$ 5$ ).

\section{Type II Supernovae}

The light curves of these supernovae have been proposed for a subclassification scheme (Barbon et al. 1979, Doggett \& Branch 1985, Young \& Branch 1989). Some events exhibit a constant brightness for several weeks after maximum light, while others decline at a more or less constant rate. This separation into plateau (II-P) and linear (II-L) light curve types has been applied to many objects (Patat et al. 1993), but in the light of the physical models it might be more appropriate to drop such a distinction and assess the length of the plateau phase which depends on the envelope mass and the explosion energy. In the following we will discuss the physical processes dominating the emission of a "generic" light curve of a type II supernova, and point out the most prominent deviations observed to date. Much of the details have been learned from SN 1987A and the reader is referred to the relevant literature.

The optical display of a type II supernova starts with the shock breaking out at the surface of the progenitor star a few hours after core collapse. Up to this moment the star looks like any evolved supergiant, since the envelope does not have enough time to adjust to the rapid changes in the core at advanced burning stages. With the initial X-ray and UV burst the light curve peaks within hours before the cooling of the atmosphere decreases the flux very rapidly. The only observations of this phase we have to date are from SN 1987A (Kirshner et al. 1987, Arnett et al. 1989) and SN 1993J (c.f. contributions at this conference). For stars with expanded envelopes (red supergiants) this phase lasts longer than for more compact progenitors (Falk \& Arnett 1977, Klein \& Chevalier 1978). The steep drop following the peak is only stopped once the photosphere is balanced by the expansion of the ejecta. This results in the plateau phase which lasts as long as the photosphere does not run into the diffusion wave formed by decay of radioactive material in the core. The length of the plateau is mainly a function of the explosion energy, and thus the expansion velocity of the ejecta, and the depth of the envelope, i.e. its mass (Chugai 1991, Hsu et al. 1993). During this phase the colors of the supernova tend to remain constant implying a constant temperature (Schmidt et al. 1992). Once the photosphere has receded deep enough to encounter the additional heating from the decay of ${ }^{56} \mathrm{Ni}$ and ${ }^{56} \mathrm{Co}$ the plateau is extended for a short time (Woosley \& Weaver 1986, Hsu et al. 1993), before the light curve starts declining at a constant rate. The transition between the plateau and the decline is determined by the decreasing optical depth to optical photons. For stars with massive envelopes all the energy released by the ${ }^{56} \mathrm{Ni}-{ }^{56} \mathrm{Co}-{ }^{56} \mathrm{Fe}$ decay chain is converted into optical emission and the bolometric light curve approaches a decline rate corresponding the life-time of ${ }^{56} \mathrm{Co}$ (Suntzeff \& Bouchet 1990, Turatto et al. 1990, Schmidt et al. 1993). The deviations of SN 1987A and SN 1993J from this general picture point to different progenitor stars. SN 1987A was the explosion of a compact blue supergiant and much of the explosion energy went into overcoming the large potential energy. Thus, the photosphere receded very rapidly and no plateau was formed. The second broad maximum in the light curve was produced 
by the heating from the ${ }^{56} \mathrm{Ni}$ and ${ }^{56} \mathrm{Co}$ decays. The large envelope mass resulted in the extended peak for this supernova. In the case of SN 1993J a rather small envelope mass prevented the formation of an extended recombination period and the second maximum was again formed by the diffusion wave from the radioactive decays. The reduced length of this peak also indicates a small envelope.

The decline rate at late times might be changed by dust formation (Lucy et al. 1991, Meikle et al. 1993) and the ionization freeze-out (Fransson \& Kosma 1993) as observed in SN 1987A. The former shifts the flux from optical wavelengths into the infrared by heating of newly formed dust grains, while the second effect arises from the fact that the time of de-excitation becomes comparable to the expansion time of the supernova and this additionally "stored" energy becomes visible. Although probably not uncommon in other supernovae, these processes have been observed only in SN 1987A where they occurred $\sim 6$ and $\sim 12$ magnitudes below peak brightness.

Eventually, energy sources other than the $\gamma$-rays from ${ }^{56} \mathrm{Co}$ become important and shape the light curve. Prime candidates are long-lived radio-isotopes like ${ }^{57} \mathrm{Co},{ }^{22} \mathrm{Na}$, and ${ }^{44} \mathrm{Ti}$ (Woosley et al. 1989), a buried pulsar loosing angular momentum and ionizing a plerion (Chevalier \& Fransson 1992), light echos (Chevalier 1986, Chugai 1992), and shock-interaction with circumstellar matter (Chevalier 1990, Chugai 1992, Chevalier \& Fransson 1994). Such changes have been observed in a few supernovae several years past explosion, most prominently in SN 1980K (Fesen \& Becker 1990, Leibundgut et al. 1991b, Uomoto 1991) where the emission has remained steady at $\mathrm{V} \sim 23 \mathrm{mag}$ for the last three years. Other supernovae with similar light curves might be SN 1979C (Fesen \& Matonick 1993) and SN 1970G (Fesen 1993). The most likely energy source in these cases is the shock heating the circumstellar material lost by the progenitor star as a stellar wind.

A group of SNe II displays remarkable deviations from the above scenario. The peaks of their light curves have never been observed and their decline rates are much smaller than what is expected from ${ }^{56}$ Co decays. The best observed examples to date are SN 1986J (Rupen et al. 1987, Leibundgut et al. 1991b), SN 1987F (Filippenko 1989), and SN 1988Z (Stathakis \& Sadler 1991, Filippenko 1991, Turatto et al. 1993). Although only SN $1988 Z$ has a well-observed light curve (Turatto et al. 1993), the slow decline rates are also reflected in the evolution of line fluxes (Filippenko 1989, Leibundgut et al. 1991b, Turatto et al. 1993). The nature of the emission and its power source are not resolved, but interaction with a very dense material close to the supernova is most likely (Filippenko 1991, Chugai and Danziger 1994). All known objects of this class are evolving very slowly. It appears as if the interactions with the pre-supernova wind dominates the light curve from the start, thus obliterating other processes.

\section{Type Ia Supernovae}

The apparent homogeneity of SNe Ia has rendered most photometric subclassification useless. The proposed schemes were either too detailed for the available data (Pskovskii 1977,1984 ) or not very meaningful (Barbon et al. 1973). The adoption of template light curves (Doggett \& Branch 1985, Leibundgut 1988) has proven helpful for the comparison of individual events, while not being a satisfactory description for some. Recently the CTIO group has pointed out some severe deviations in the light curve shapes among well-observed SNe Ia (Phillips 1993, Suntzeff, this volume). They consist in different decline rates after maximum, variations in the time of maximum in different filters, and different shapes altogether - especially for the near-infrared light curves (Frogel et al. 1987, Suntzeff, this volume).

The physics of SNe Ia light curves has been discussed in several recent publications 


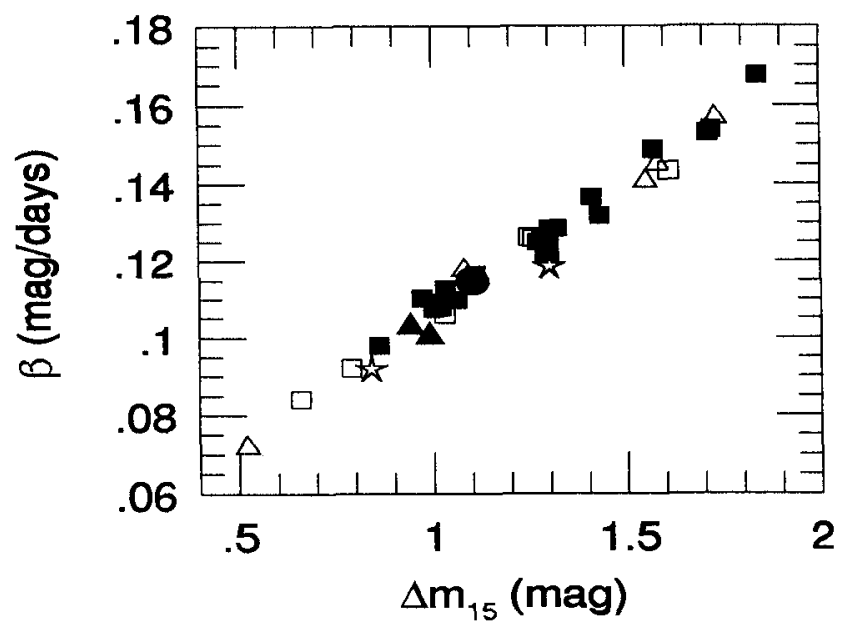

Figure 1. Decline rates vs. decline for the first 15 days past maximum. Filled symbols are for $\mathrm{B}$ data, while open symbols designate pg data. The squares are $\mathrm{SNe} \mathrm{Ia}$ and the triangles $\mathrm{SNe} I$. The filled dot is for the template light curve. Two SNeIb/c are displayed as stars.

(Höflich et al. 1993, Khokhlov et al. 1993, Leibundgut \& Pinto 1992). Although a completely different explosion mechanism is at work, the light curves are shaped not unlike certain phases of SNe II. Two main periods, determined by the optical depth for optical photons in the ejecta, can easily be distinguished: the peak phase, where the energy is diffusing out of the explosion, and the rapid decline after $\sim 40$ days past maximum, when the optically thin nebula is cooled by line emission. While this simple description has been valid for the U, B, and V light curves a distinctive "second peak" is observed in the J, H, and K light curves (Elias et al. 1981, 1985), and is also obvious in well-sampled $\mathrm{R}$ and I light curves (Suntzeff, this volume). The apparent flux redistribution remains unexplained to date.

Severe photometric disparities among SNeIa have been observed recently. A large range in decline rates after maximum was found by Phillips (1993). To step beyond the template approach a fitting procedure was adopted by Vacca \& Leibundgut (1994) that describes the UBV light curves as a gaussian atop a line to match the peak and late decline periods, respectively. This scheme allows to fit individual light curves to the available photometry of SNe Ia in an objective manner. The decline rates, as measured halfway down the initial decline of the light curves, are found to range from $\sim 0.07 \mathrm{mag} /$ day to $\sim 0.17 \mathrm{mag} /$ day and to correlate extremely well with the decline during the first 15 days past maximum as defined by Phillips (1993). Figure 1 displays this correlation for 32 $\mathrm{SNeIa}$ and $8 \mathrm{SNeI}$ (for which no subclassification was available). This vindicates the notion that there is an appreciable range of light curve shapes among SNe Ia.

The fitting procedure determines a number of additional parameters, such as, the peak magnitude, the time of maximum, the slope of the late-time decline, the magnitude drop until the inflection, and the time between maximum date and the inflection. The inflection is a well-defined parameter as it can be chosen to be some fraction of the contribution of the gaussian to the total flux ( $10 \%$ was adopted). Note that these parameters are defined by a mathematical model of the brightness evolution with a least squares procedure and are defined differently than the ones proposed by Pskovskii $(1977,1984)$. The fixed-shape template curves of Leibundgut (1988) agrees in general with the mean 


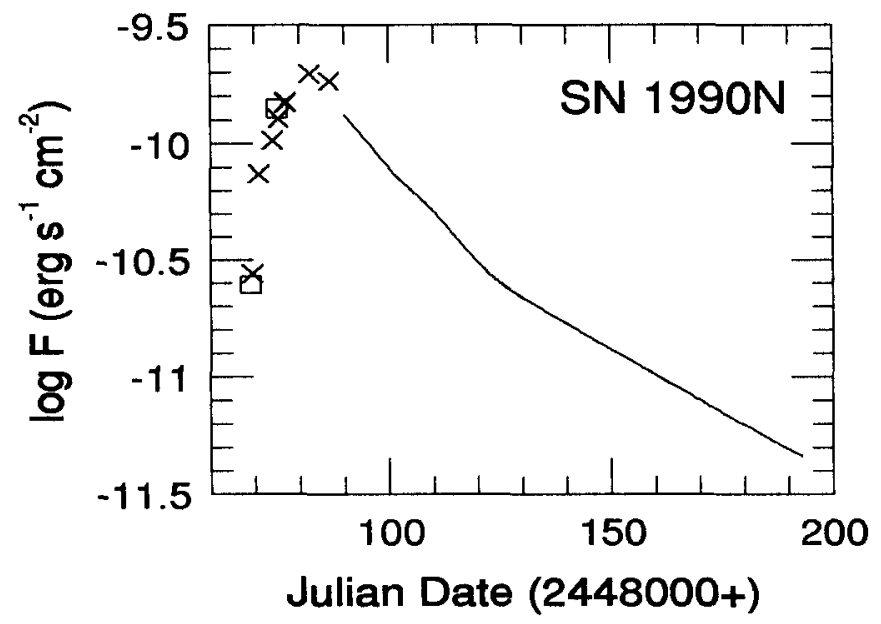

FIGURE 2. The bolometric light curve of SN 1990N. The crosses are UV observations added to the optical fluxes. The squares are direct integrations of UV and optical spectra. The solid line delineates the bolometric light curve derived from the templates

of the distributions of the parameters, but does not account for the observed range in the parameters.

Bolometric light curves for SNe Ia have been rare to date. Leibundgut \& Pinto (1992) published a luminosity evolution based on the UBVJHK templates for SNe Ia. The biggest deficiency is the lack of $\mathrm{R}$ and I filter photometry and hence the large interpolation in the integration over the wavelengths. This is remedied by the superb data now becoming available (Suntzeff, this volume). Sufficient UV data is needed to determine the peak and are provided by the series of IUE spectra of SN 1990N (Leibundgut et al. 1991a) and IUE and HST spectra of SN 1992A (Kirshner et al. 1993). The UV contribution is $\sim 10 \%$ of the total at the UV peak, but has an important effect on the date of maximum. The lack of infrared data during the maximum phase is unimportant since the fraction of the infrared flux is $<5 \%$ at 5 days past $B$ maximum and increases only later, while the contribution of the UV decreases after peak as the supernova becomes cooler. In Figure 2 we present the composite bolometric light curve of SN 1990N which includes the maximum. The bolometric light curve peaks approximately 2 to 3 days before B and, with the limits presented in Leibundgut et al. (1991a), we find a rise time of the bolometric light curve between 14 and 18 days. Only the slowest deflagration and delayed-detonation models straddle the lower end of this range (Khokhlov et al. 1993).

\section{Supernovae Type Ib/c}

The photometry of SNe Ib and SNe Ic has been buried among the data of SNe Ia for a long time. Very few light curves of these objects are available (c.f. Porter \& Filippenko 1987, Swartz \& Wheeler 1991, Kirshner 1990). Most of these are sampled poorly so that direct comparisons are difficult. For example, Vacca \& Leibundgut (1994) find only two $\mathrm{SNe} \mathrm{Ib} / \mathrm{c}$ with sufficient $\mathrm{B} / \mathrm{pg}$ photometry to fit their model curves. A difference in the decline rates at late epochs between $\mathrm{SNe} \mathrm{Ib}$ and $\mathrm{SNe}$ Ic has been suggested (Swartz \& Wheeler 1991), but has yet to be confirmed with accurate photometry.

The physics of SNe Ib/c light curves is very similar to that of SNeII, since they are presumably also core collapse objects. The peak from the breakout is reduced due to the compact nature of the progenitor and the absence of a substantial envelope (Ensman 
\& Woosley 1988, Nomoto et al. 1990) which also suppresses an extended recombination phase in the light curves. A broad peak is formed much like in SN 1987A and SNe Ia by the diffusion wave. An important diagnostic for the envelope masses will be the decline rates at late times. The light curve of SN $1993 \mathrm{~J}$ is probably much like a type $\mathrm{Ib} / \mathrm{c}$ light curve (Wheeler \& Filippenko, this volume).

\section{Conclusions}

The last few years have seen appreciable progress in integrating observations and models. Bright supernovae with an abundance of data have allowed to improve models and develop some of their details. Light curves have been a mainstay for this advance. Bolometric light curves represent the most direct comparison between models and observations. Their construction for SNe II has been eased by improved hydrodynamic calculations that provide "bolometric corrections." The individuality of these supernovae will further increase the range of different characteristics of these explosions and their progenitor stars.

The photometric homogeneity of SNeIa is put to test with the improved $\mathrm{R}$ and $\mathrm{I}$ light curves now obtained regularly. Eventually the dominance of $\mathrm{B}$ and $\mathrm{V}$ data will be replaced by a panchromatic view, and much needed bolometric light curves for individual objects will become available. Whether there exists a large fraction of SNe Ia with very similar behavior, or whether the class stretches evenly across the observed range, will only be answered with good light curves.

$\mathrm{SNe} \mathrm{Ib} / \mathrm{c}$ are photometrically still indistinguishable from SNe Ia in the optical, although they are physically probably more related to SNe II. More data is needed for support of modeling efforts and SN 1993J is a promising candidate for a study of such events.

No attention has been given to external effects which can change the shape of light curves. Background contamination can be a severe problem (Boisseau \& Wheeler 1991), which, however, can be tackled with modern photometry programs. Absorption and reddening plague the discussion of homogeneity and the determination of absolute luminosities. They also can change the shape of the light curve due to the strong color evolution of supernovae (Leibundgut 1988). Differences in filter passbands can cause confusion (Menzies 1989, Hamuy et al. 1990). Finally, cosmological redshifts affect the light curves of very distant SNe (Hamuy et al. 1993).

The combination of photometry and spectroscopy remains a very powerful tool for the analysis of supernovae. With both at hand the progress in our understanding of these fireworks in the universe will be ensured.

\section{Acknowledgements}

I am indebted to J. Hsu, P. Pinto, and W. Vacca for many helpful discussions of supernova light curves. Financial support from the Swiss National Science Foundation and NSF grant AST-9115174 are acknowledged.

\section{REFERENCES}

Arnett, W. D., Bahcall, J. N., Kirshner, R. P., \& Woosley, S. E. 1989, ARA\& A 27, 629

Barbon, R., Ciatti, F., \& Rosino, L. 1973, A\& A 25, 65

Barbon, R., Ciatti, F., \& Rosino, L. 1979, A\& A 72, 287

Boisseau, J. R. \& Wheeler, J. C. 1991, AJ 101, 1281.

Bouchet, P., Danziger, I. J., \& Lucy, L. B. 1991, AJ, 102, 1135 
Chevalier, R. A. 1986, ApJ 308, 225

Chevalier, R. A. 1990, Supernovae, ed. A. G. Petschek (Springer-Verlag, New York), p. 91

Chevalier, R. A. \& Fransson, C. 1992, ApJ 395, 540.

Chevalier, R. A. \& Fransson, C. 1994, ApJ, 420, 268

Chugai, N. N. 1991, Sov. Ast. Lett. 17, 210

Chugai, N. N. 1992, Sov. Ast. 36, 63

Chugai, N. N. \& Danziger, I. J. 1994, MNRAS, 268, 173.

Doggett, J. B. \& Branch, D. 1985, AJ 90, 2303

Elias, J. H., Frogel, J. A., Hackwell, J. A., \& Persson, S. E. 1981, ApJ, 251, L 13

Elias, J. H., Matthews, K., Neugebauer, G., \& Persson, S. E. 1985, ApJ, 196, 379.

Ensman, L. M. \& Woosley, S. E. 1988, ApJ, 333, 754.

Falk, S. W. \& Arnett, W. D. 1977, ApJS, 33, 515.

Fesen, R. A. 1993, ApJ, 413, L109

Fesen, R. A. \& Becker, R. H. 1990, ApJ, 351, 437

Fesen, R. A. \& Matonick, D. M. 1993, ApJ, 407, 110

Filippenko, A. V. 1989, AJ, 97, 726

Filippenko, A. V. 1991, SN 1987A and Other Supernovae, eds. I. J. Danziger \& K. Kjär (ESO, Garching), p. 343

Fransson, C. \& Kosma, C. 1993, ApJ, 408, L25

Frogel, J. A., Gregory, B., Kawara, K., Laney, D., Phillips, M. M., Terndrup, D., Vrba, F., \& Whitford, A. E. 1987, ApJ, 315, L129

Hamuy, M., Suntzeff, N. B., Bravo, J., \& Phillips, M. M. 1990, PASP, 102, 888

Hamuy, M., Phillips, M. M., Wells, L. A., \& Maza, J. 1993, PASP, 105, 787

Höflich, P., Khokhlov, A., \& Müller, E. 1993, A\& A, 268, 570

Hsu, J. J. L., Joss, P. C., Ross, R. R., \& Podsiadlowski, P. 1994, Nature 364, 509

Khokhlov, A., Müller, E., \& Höflich, P. 1993, A\& A, 270, 223

Kirshner, R. P. 1990, Supernovae, ed. A. G. Petschek (Springer-Verlag, New York), p. 59

Kirshner, R. P., et al. 1993, ApJ, 415, 589

Kirshner, R. P., Sonneborn, G., Crenshaw, D. M., \& Nassiopoulos, G. E. 1987, ApJ, 320, 602

Klein, R. I. \& Chevalier, R. A. 1978, ApJ 223, L109

Leibundgut, B. 1988, Ph.D. Thesis, University of Basel

Leibundgut, B., Kirshner, R. P., Filippenko, A. V., Shields, J. C., Foltz, C. B., Phillips, M. M., \& Sonneborn, G. 1991a, ApJ, 371, L23

Leibundgut, B., Kirshner, R. P., Pinto, P. A., Rupen, M. P., Smith, R. C., Gunn, J. E., \& Schneider, D. P. 1991b, ApJ, 372, 531

Leibundgut, B. \& Pinto, P. A. 1992, ApJ, 401, 49

Leibundgut, B., Tammann, G. A., Cadonau, R., \& Cerrito, D. 1991c, A\& AS, 89, 537

Lucy, L. B., Danziger, I. J., Gouiffes, C., \& Bouchet, P. 1991, Supernovae, ed. S. E. Woosley (Springer-Verlag, New York), p. 82

McCray, R. 1993, ARA\& A, 31, 175

Meikle, W. P. S., Spyromilio, J., Allen, D. A., Varani, G.-F., \& Cumming, R. J. 1993, MNRAS, 261,535

Menzies, J. W. 1989, MNRAS, 237, 21p

Minkowski, R. (1 964, ARA \& A, 2, 247

Nomoto, K., Filippenko, A. V., \& Shigeyama, T. 1990, A\& A, 240, Ll

Patat, F., Barbon, R., Cappellaro, R., \& Turatto, M. 1993, A\& AS, 98, 443

Phillips, M. M. 1993, ApJ, 413, L105 
Porter, A. C. \& Filippenko, A. V. 1987, AJ, 93, 1372

Pskovskii, Y. P. 1977, Soviet Ast., 21, 675

Pskovskii, Y. P. 1984, Soviet Ast., 28, 658

Rupen, M. P., van Gorkom, J. H., Knapp, G. R., Gunn, J. E., \& Schneider, D. P. 1987, AJ, 94, 61

Schmidt, B. P., Kirshner, R. P., \& Eastman, R. G. 1992, ApJ, 395, 366

Schmidt, B. P., et al. 1993, AJ, 105, 2236

Stathakis, R. A. \& Sadler, E. M. 1991, MNRAS, 250, 786

Suntzeff, N. B. \& Bouchet, P. 1990, AJ, 99, 650

Suntzeff, N. B., Phillips, M. M., Depoy, D. L., Elias, J. H., \& Walker, A. R. 1991, AJ, 102, 1118

Swartz, D. A. \& Wheeler, J. C. 1991, ApJ, 379, L13

Turatto, M., Cappellaro, E., Barbon, R., Della Valle, M., Ortolani, S., \& Rosino, L. 1990, AJ, 100,771

Turatto, M., Cappellaro, E., Danziger, I. J., Benetti, S., Gouiffes, C., \& Della Valle, M. 1993, MNRAS, 262, 128

Uomoto, A. 1991, AJ, 101, 1275

Vacca, W. D. \& Leibundgut, B. 1994, ApJ, in preparation.

Wheeler, J. C. \& Harkness, R. P. 1990, Rep. Prog. Phys., 53, 1467

Woosley, S. E., Pinto, P. A., \& Ensman, L. M. 1988, ApJ, 324, 466

Woosley, S. E., Pinto, P. A., \& Hartmann, D. 1989, ApJ, 346, 395

Woosley, S. E. \& Weaver, T. A. 1986, ARA\& A, 24, 205

Young, T.R. \& Branch, D. 1989, ApJ, 342, L79 